\title{
A DEFORMATION BOUNDING THEOREM FOR FLOW-LAW PLASTICITY*
}

\author{
By PHILIP G. HODGE, JR. (Illinois Institute of Technology, Chicago, Illinois)
}

\begin{abstract}
It is shown that the elastic principle of minimum complementary energy can be generalized to provide a bounding theorem for an elastic/perfectly-plastic material. For certain special classes of problems the bounding principle becomes a true minimum principle.

1. Introduction. A minimum theorem in continuum mechanics proves that among all members of a certain class, the complete solution to a given boundary value problem minimizes a certain functional. A bounding theorem, on the other hand, proves that the value of the functional associated with any member of a given class is not less than the value associated with the complete solution. Obviously, all minimum theorems are bounding theorems, but the converse is true if and only if the complete solution is a member of a given class.

The principles of minimum potential or complementary energy in elasticity and the theorems of limit analysis in plasticity are examples of minimum theorems, whereas recent work by Martin ${ }^{1}$ has used a class of static solutions in proving bounding theorems for dynamic problems. The present paper proves a bounding theorem for a functional of the total deformation of an elastic/perfectly-plastic material with a flow-law type of constitutive equation.

We consider the usual type of boundary value problem in which we are given a region $V$ bounded by a surface $S$. The entire surface is assumed to be composed of two types of sub-surfaces: $S_{D}$ on which either the displacement vector $\mathbf{u}$ is prescribed or the traction $\mathrm{T}$ vanishes, and $S_{T}$ on which either $\mathrm{T}$ is prescribed or $\mathbf{u}=0$. In addition the body force $\mathrm{f}$ is given throughout $V$.

If the material in $V$ is elastic, then for any stress state $\delta$ we may define the complementary energy density

$$
U_{c}(\boldsymbol{\delta})=\int \varepsilon(\boldsymbol{\delta}) \cdot d \boldsymbol{\delta}
$$

and the total complementary energy by

$$
\Pi_{c}=\int_{V} U_{c}-\int_{S_{D}} \mathbf{T} \cdot \mathbf{u} .
$$

The well-known theorem of minimum complementary energy then states that among all equilibrium states of stress the actual one minimizes $\Pi_{c}$.

For an elastic/perfectly-plastic material, the theorem is, in general, not valid. The purpose of the present note is to point out that by placing certain additional re-

${ }^{*}$ Received August 23, 1965; revised manuscript received November 22, 1965. This investigation was supported by the Office of Naval Research.

1J. B. Martin: A displacement bound principle for inelastic continua subjected to certain classes of dynamic loading, J. Appl. Mech 32, 1-6 (1965); A displacement bound technique for elastic continua subjected to a certain class of dynamic loading, J. Mech. Phys. Sol. 12, 165-175 (1964) 
strictions on the class of admissible stress states, a slightly weaker theorem may be proved, and that for some special problems the original theorem is valid. A similar but weaker conclusion is reached concerning the theorem of minimum potential energy.

2. Statement and proof of the bounding complementary energy theorem. Rather than introduce seemingly unmotivated restrictions at the outset, we shall formally attempt to prove the principle of minimum complementary energy for an elastic/perfectlyplastic material. At a certain point in the attempted proof it will then become evident what additional restriction is necessary for the desired conclusion to be valid.

The behavior of an elastic/perfectly-plastic material is defined by the yield inequality

$$
f(\boldsymbol{d}) \leq 0,
$$

where $f$ is a convex function, and by the flow law

$$
\boldsymbol{\varepsilon}^{\prime}=\mathbf{c} \cdot \boldsymbol{\sigma}^{\prime}+\boldsymbol{\varepsilon}_{p}^{\prime},
$$

where primes indicate differentiation with respect to time. Here $\mathbf{c}$ is the fourth order tensor of elastic constants. The plastic strain-rate tensor $\varepsilon_{p}^{\prime}$ must vanish if $f<0$ or $f^{\prime}<0$; otherwise it must be directed along a normal to the surface $f=0$.

Given the stress $\delta$ at a point for all times $\tau, 0 \leq \tau \leq t$, one can formally integrate (4) to obtain

$$
\varepsilon=\mathbf{c} \cdot \boldsymbol{\sigma}+\int_{0}^{t} \varepsilon_{p}^{\prime} d \tau .
$$

Since $\boldsymbol{\varepsilon}_{p}^{\prime}=0$ when $f<0$, the integration in Eq. (5) effectively takes place only during time intervals in which the point is plastic. Substituting the flow law (4) into (1) and integrating by parts, we obtain

$$
U_{c}(\boldsymbol{\sigma})=\frac{1}{2} \boldsymbol{\sigma} \cdot \mathbf{c} \cdot \boldsymbol{\sigma}+\int_{0}^{t}[\boldsymbol{\sigma}(t)-\boldsymbol{\sigma}(\tau)] \cdot \epsilon_{p}^{\prime}(\tau) d \tau .
$$

Now, let $\mathbf{\sigma}^{\circ}(\tau)$ be a stress state which satisfies internal equilibrium, all stress boundary conditions, and the yield inequality (3) for all $0 \leq \tau \leq t$. Use the definition of plastic strain rate to define a related $\varepsilon_{p}^{0^{\prime}}$. Equation (6) can then be used to define

$$
U_{c}\left(\boldsymbol{\sigma}^{0}\right)=\frac{1}{2} \boldsymbol{\sigma}^{0} \cdot \mathbf{c} \cdot \boldsymbol{\sigma}^{0}+\int_{0}^{t}\left[\boldsymbol{\sigma}^{0}(t)-\boldsymbol{\sigma}^{\prime \prime}(\tau)\right] \cdot \varepsilon_{p}^{n^{\prime \prime}}(\tau) d \tau .
$$

Next (2) can be used to define the total complementary energy for the statically admissible state $\boldsymbol{\sigma}^{\mathbf{0}}, \boldsymbol{\varepsilon}^{\mathbf{0}}$ and for the actual solution $\boldsymbol{\sigma}, \boldsymbol{\varepsilon}$. Using the principle of virtual work we can then write the difference

$$
\Delta \Pi_{c} \equiv \Pi_{c}\left(\boldsymbol{\delta}^{n}\right)-\Pi_{c}(\boldsymbol{\delta})=\int_{V}\left[U_{c}\left(\boldsymbol{\delta}^{0}\right)-U_{c}(\boldsymbol{\delta})-\left(\boldsymbol{\delta}^{0}-\boldsymbol{\delta}\right) \cdot \varepsilon\right]
$$

The principle of minimum complementary energy will be true if and only if it can be shown that $\Delta \Pi_{c} \geq 0$ for all statically admissible stress states.

Using (5-7) and the symmetry of $\mathbf{c}$, we easily obtain

$$
\begin{aligned}
\Delta \mathrm{II}_{\boldsymbol{c}}=\frac{1}{2} \int_{V}\left(\boldsymbol{\sigma}^{0}-\boldsymbol{\sigma}\right) \cdot \mathbf{c} \cdot\left(\boldsymbol{\sigma}^{0}-\boldsymbol{\sigma}\right) \\
\quad+\int_{V} \int_{0}^{t}\left[\mathbf{\sigma}^{0}(t)-\boldsymbol{\sigma}^{0}(\tau)\right] \cdot \epsilon_{\nu}^{0^{\prime}}(\tau) d \tau+\int_{V} \int_{0}^{t}\left[\boldsymbol{\sigma}(\tau)-\boldsymbol{\sigma}^{0}(t)\right] \cdot \boldsymbol{\varepsilon}_{\nu}^{\prime}(\tau) d \tau .
\end{aligned}
$$


Since $\mathbf{c}$ is positive definite, the first integral in (9) is non-negative but may be arbitrarily close to zero for $\mathbf{d}^{0}$ close to $\mathbf{o}$.

At any time $\tau$, either $\boldsymbol{\varepsilon}_{p}^{\prime}(\tau)$ vanishes, or $\boldsymbol{\sigma}(\tau)$ is on the surface $f=0$ and $\boldsymbol{\varepsilon}_{p}^{\prime}(\tau)$ is directed along an outward normal to the surface at that point. Since $\boldsymbol{\delta}^{0}(t)$ is on or interior to the surface $f=0$ and since $f$ is convex, the third integrand of (9) is non-negative for all $0<\tau<t$, and hence the integral is non-negative.

Since the first and third terms are non-negative but may be arbitrarily close to zero for properly chosen statically admissible states, the theorem will be valid if and only if the second term is non-negative. However, the convexity argument used on the third term shows that the second term is non-positive. Therefore, the theorem is generally false.

We note, however, that the term in question depends only upon the statically admissible state for which we have complete freedom of choice, and not upon the actual solution which must be regarded as known. Therefore, if we define a class of restricted statically admissible states which satisfy all of the requirements of a statically admissible state plus the requirement that for all $0<\tau<t$

$$
\left[\boldsymbol{\sigma}^{0}(t)-\boldsymbol{\sigma}^{0}(\tau)\right] \cdot \varepsilon_{p}^{0^{\prime}}(\tau)=0,
$$

then the following theorem is obviously valid:

Theorem of Bounding Complementary Energy (Statement 1): The total complementary energy $\Pi_{c}^{0}$ of any restricted statically admissible state is an upper bound on the total complementary energy of the actual solution.

3. Discussion. We observe first that in general the actual solution will not satisfy the restriction (10). Therefore it will not be a member of the class of restricted statically admissible states so that the least upper bound provided by the theorem may still be greater than $\Pi_{c}$ for the actual state. A consequence of this limitation is that the theorem cannot be used in the derivation of equations, since it is not in any sense a variational principle.

Substitution of the restriction (10) into (7) shows that

$$
U_{c}\left(\mathbf{\sigma}^{0}\right)=\frac{1}{2} \mathbf{\sigma}^{0} \cdot \mathbf{c} \cdot \mathbf{\sigma}^{0} .
$$

This is precisely the elastic complementary energy density, so that the theorem may be given an alternative statement:

Theorem of Bounding Complementary Energy (Statement 2): If, for any stress state which satisfies equilibrium, stress boundary conditions, and the yield inequality, the total complementary energy is calculated on the basis of an elastic material, the result will be an upper bound on the total complementary energy of the elastic/perfectlyplastic material.

For certain restricted classes of problems, the actual solution may satisfy the restriction (10). For such problems the principle is a true minimum principle.

An obvious such special case is the elastic material for which $\varepsilon_{p}^{\prime}=0$. Thus the well known elastic principle of minimum complementary energy is a special case of the present theorem.

A second special case is one for which whenever $\varepsilon_{p}^{\prime} \neq 0, \boldsymbol{\sigma}$ is a constant in time. This will occur if the plastic stress distribution is statically determinate so that there is a unique state of stress which satisfies equilibrium, stress boundary conditions, and the yield equality $f=0$, and if the loading is such that once a material point becomes 
plastic it never unloads. One-dimensional problems such as trusses and beams and frames under bending belong in this category provided that no plastic point ever unloads. A less trivial example is torsion of a bar of simply connected cross-section under a monotonic torque for which the plastic stress solution is given by the Nadai sand-hill analogy.

As a third special case we regain the minimum principle for piecewise-linear plasticity with regular progression ${ }^{2}$. If the actual stress point is always on the same linear side of a yield surface, then $\boldsymbol{\sigma}(t)-\boldsymbol{\sigma}(\tau)$ is perpendicular to $\boldsymbol{\varepsilon}_{p}^{\prime}(\tau)$; if it is in a corner $\boldsymbol{\sigma}(t)=\boldsymbol{\sigma}(\tau)$; finally, if it moves from a side to a corner (10) is also satisfied. However, although the actual solution must progress regularly for the minimum principle to hold, the bounding theorem is valid provided only that the comparison solution is a regular progression. This fact does not appear to have been recognized earlier ${ }^{2}$.

The obvious question to ask next is whether a similar generalization can be made for the principle of minimum potential energy. Unfortunately, the conclusions are much less satisfactory here.

Using the definitions

$$
\begin{aligned}
& U(\varepsilon)=\int \boldsymbol{\delta}(\varepsilon) \cdot d \varepsilon, \\
& \Pi(\varepsilon)=\int_{V} U(\varepsilon)-\int_{S T} \mathbf{T} \cdot \mathbf{u}-\int_{V} \mathbf{f} \cdot \mathbf{u},
\end{aligned}
$$

one easily obtains the analogue to (9):

$$
\begin{aligned}
\Delta \Pi=\frac{1}{2} \int_{V}\left(\boldsymbol{\sigma}^{*}-\boldsymbol{\sigma}\right) \cdot \mathbf{c} \cdot\left(\boldsymbol{\sigma}^{*}-\boldsymbol{\sigma}\right) \\
\quad+\int_{V} \int_{0}^{t}\left[\boldsymbol{\sigma}^{*}(\tau)-\boldsymbol{\sigma}(t)\right] \cdot \varepsilon_{p}^{* \prime}(\tau) d \tau+\int_{V} \int_{0}^{t}[\boldsymbol{\sigma}(t)-\boldsymbol{\sigma}(\tau)] \cdot \varepsilon_{p}^{\prime}(\tau) d \tau .
\end{aligned}
$$

Here ( )* refers to a kinematically admissible field for which the strains and displacements satisfy compatibility and displacement boundary conditions, and the stresses are determined by the flow law and yield inequality. The first term of (13) is positive definite, the second is non-negative from the convexity of $f$, and the third is non-positive and hence must vanish for $\Delta \Pi$ to be necessarily non-negative. Thus the theorem will be valid only if

$$
[\boldsymbol{\sigma}(t)-\boldsymbol{\sigma}(\tau)] \cdot \boldsymbol{\varepsilon}_{p}^{\prime}(\tau)=0
$$

Equation (14) depends on the actual state, and hence is not subject to manipulation. Therefore, for those special cases discussed in the preceding section for which the actual state satisfies (14), the principle of minimum potential energy is valid. However, for the general case of an elastic/perfectly-plastic material the principle is not valid, nor can it be used with restricted admissible functions to provide bounds on the potential energy. 